\title{
The Role of USP18 in Insulin Resistance
}

\section{Anjing Zhu, Limin Chen and Yujia Li*}

Chinese Academy of Medical Sciences and Peking Union Medical College, Institute of Blood Transfusion, Key Laboratory for Transfusion-transmitted Infectious Diseases of Sichuan Province, Chengdu, China

\section{Brief Introduction to USP18}

Host innate immunity is regarded as the first line of defense to fight against virus infections. Type I Interferon (IFN) is one of the main components in the innate immune response [1]. Type I and III IFNs function through activating the Jak/STAT signaling pathway and then up-regulating the expression levels of hundreds of interferon stimulated genes(ISGs) [2,3]. Many ISGs, including ubiquitin-specific protease 18 (USP18, also known as UBP43), exert important antiviral and/or immuno-modulatory effects in different stages of virus infection [4].

USP18 is a member of the deubiquitinating enzyme family [5], which removes ISG15 from its conjugated proteins (deISGylation) [6]. As a negative regulator of type I interferon signaling pathway [7], USP18 binds to the type interferon receptor sub-unit 2 (IFNAR2) to prevent JAK1 phosphorylation and thereby inhibit the type I/III IFN signaling pathways [8].

\section{Insulin Resistance}

The rates of insulin-mediated glucose disposal are varied in different populations, mainly due to adiposity, fitness or inheritance [9]. When the host's response to insulin decreases, which is named as insulin resistance (IR), insulin concentration will increase (hyperinsulinemia) to keep blood glucose level normal. Once the blood glucose derails during hyperinsulinemia, diabetes occur [10]. IR plays a fundamental role in the pathogenesis of type 2 diabetes mellitus (T2DM). In addition, IR is also related to other diseases, such as Alzheimer's disease, endometrial cancer, and inflammation $[11,12]$.

\section{The Role of USP18 in Insulin Resistance}

USP18 alleviates insulin resistance through its deubiquitinating activity. Shimin et al. observed decreased expression levels of USP18 in the livers of non-alcoholic steatohepatitis (NASH) patients and obese mice [13]. This interesting observation indicates that USP18 is related to hepatic steatosis and glucose metabolism. In addition, the authors studied the function of USP18 in the obese mice through transgene and knockout technology. USP18 over-expression rescued insulin resistance and inflammation. On the other hand, USP18 knockout (deficiency) aggravated hepatic steatosis, insulin resistance and inflammation. Mechanistically, the authors focused their study on the upstream regulator, transforming growth factor $\beta$-activated kinase 1(TAK1), which was essential for USP18 to regulate hepatic steatosis, insulin resistance, and inflammation. They demonstrated that USP18 inhibits TAK1 activation through its deubiquitinating activity to suppress the downstream JNK and NF-KB signaling pathways. As a result, the USP18-TAK1-JNK1 axis balanced Ser/Tyr-IRS1 phosphorylation to regulate insulin sensitivity in hepatocytes. Therefore, USP18 could be a potential therapeutic target for NASH patients [13]. Furthermore, USP18 was found to directly interact with insulin receptor substrate4(IRS4), which implied that USP18 may play an important role in insulin signaling pathway although the detailed mechanism remains to be determined [14].

\section{Conclusion}

Previously, many people thought virus infection has nothing to do with diabetes mellitus (DM). However, when people found that the diabetes mellitus was related to inflammation, the association between virus infection and DM drew a lot of attention. There is a close link between USP 18 and DM as shown by the most recent study that USP 18 could alleviate insulin resistance through its deubiquitinating activity. The role of USP18 induction following virus infection in IR and its underlying mechanisms remain to be determined.

\section{Reference}

1. Joo SS, Won TJ, Kim MJ, Hwang KW, Lee DI (2006) Interferon signal transduction of biphenyl dimethyl dicarboxylate/amantadine and anti-HBV activity in HepG 2 2.2.15. Arch Pharm Res 29: 405-411.

2. de Veer MJ, Holko M, Frevel M, Walker E, Der S, Paranjape JM, et al. (2001) Functional classification of interferon-stimulated genes identified using microarrays. J Leukoc Biol 69: 912-920.

3. Sadler AJ, Williams BR (2008) Interferon-inducible antiviral effectors. Nat Rev Immunol 8: 559-568.

4. Wong MT, Chen SS (2016) Emerging roles of interferon-stimulated genes in the innate immune response to hepatitis $C$ virus infection. Cell Mol Immunol 13: 11-35.

5. Liu LQ, Ilaria R Jr, Kingsley PD, Iwama A, van Etten RA, et al. (1999) A novel ubiquitin-specific protease, UBP43, cloned from leukemia fusion protein AML1ETO-expressing mice, functions in hematopoietic cell differentiation. Mol Cell Biol 19: 3029-3038

6. Ritchie KJ, Zhang DE (2004) ISG15: The immunological kin of ubiquitin. Semin Cell Dev Biol 15: 237-246.

7. Francois-Newton V, Magno de Freitas Almeida G, Payelle-Brogard B, Monneron $D$, Pichard-Garcia L, et al. (2011) USP18-based negative feedback control is induced by type I and type III interferons and specifically inactivates interferon alpha response. PLoS One 6: e22200.

8. Malakhova OA, Kim KI, Luo JK, Zou W, Kumar KG, et al. (2006) UBP43 is a novel regulator of interferon signaling independent of its ISG15 isopeptidase activity. Embo J 25: 2358-2367.

9. Yeni-Komshian H, Carantoni M, Abbasi F, Reaven GM (2000) Relationship between several surrogate estimates of insulin resistance and quantification of insulin-mediated glucose disposal in $\mathbf{4 9 0}$ healthy nondiabetic volunteers. Diabetes Care 23: 171-175.

10. Kahn CR (1978) Insulin resistance, insulin insensitivity, and insulin unresponsiveness: A necessary distinction. Metabolism 27: 1893-1902.

11. Ma L, Wang J, Li Y (2015) Insulin resistance and cognitive dysfunction. Clin Chim Acta 444: 18-23.

*Corresponding author: Yujia Li, Chinese Academy of Medical Sciences and Peking Union Medical College, Institute of Blood Transfusion, Key Laboratory for Transfusion-transmitted Infectious Diseases of Sichuan Province, Chengdu, China, Tel: 86-28-61648530; E-mail: lily83630@163.com

Received October 20, 2018; Accepted October 22, 2018; Published October 29, 2018

Citation: Zhu A, Chen L, Li Y (2018) The Role of USP18 in Insulin Resistance. J Bioanal Biomed 10: e158. doi:10.4172/1948-593X.1000e158

Copyright: @2018 Zhu A, et al. This is an open-access article distributed under the terms of the Creative Commons Attribution License, which permits unrestricted use, distribution, and reproduction in any medium, provided the original author and source are credited. 
12. Mu N, Zhu Y, Wang $Y$, Zhang $H$, Xue $F(2012)$ Insulin resistance: A significant risk factor of endometrial cancer. Gynecol Oncol 125: 751-757.

13. An S, Zhao LP, Shen LJ, Wang S, Zhang K, et al.(2017) USP18 protects against hepatic steatosis and insulin resistance through its deubiquitinating activity. Hepatology 66: 1866-1884.

14. Jiao B, Shi X, Chen Y, Ye H, Yao M, et al. (2017) Insulin receptor substrate-4 interacts with ubiquitin-specific protease 18 to activate the Jak/STAT signaling pathway. Oncotarget 8: 105923-105935 